Diskussion um Ginkgopräparate

\title{
Können Sie den geistigen Abbau aufhalten?
}

- Ist eine Therapie mit Ginkgo biloba in der Lage, bei älteren Menschen den Abbau der kognitiven Leistungsfähigkeit hinauszuzögern? In einer kürzlich publizierten 6-Jahres-Studie aus den USA war dies nicht der Fall. Doch eine Metaanalyse kommt zu einem anderen Ergebnis.

An der placebokontrollierten US-Studie hatten sich 3100 Menschen im Alter von 72-96 Jahren beteiligt. Fünf von sechs Teilnehmern waren kognitiv gesund, die Übrigen hatten bereits leichte kognitive Einschränkungen. Durch eine im Schnitt sechs Jahre dauernde Ginkgobehandlung wurde weder die Entwicklung einer Demenz verhindert noch der Verlust kognitiver Leistungen verzögert. Allerdings war auch in der Placebogruppe der geistige Abbau außergewöhnlich gering. Das könnte den fehlenden Effekt der Ginkgobehandlung möglicherweise erklären, wie es in einer Stellungnahme des Arzneimittelunternehmens Schwabe heißt.

Unterstützt wird diese Vermutung durch eine ebenfalls kürzlich publizierte Metaanalyse von 29 Studien mit 2141 Teilnehmern. Die Einnahme von Ginkgo-biloba-Extrakten kann danach nicht nur das Gedächtnis verbessern, sondern auch in anderen Funktionsbereichen die geistige
Leistungsfähigkeit erhöhen. In die Metaanalyse gingen alle verfügbaren, placebokontrollierten Doppelblindstudien aus den Jahren 1980 bis 2007 ein, in denen über mindestens vier Wochen spezifische Gehirnleistungsfunktionen unter Ginkgoextrakten untersucht worden waren. Studienteilnehmer waren Personen mit nachlassender mentaler Leistungsfähigkeit und gesunde Menschen.

\section{Verbesserung von komplexen kognitiven Funktionen}

Im Bereich Gedächtnis wiesen 21-33\% der Tests ein signifkant positives Ergebnis zugunsten von Ginkgo auf. Das ist vier- bis sechsmal mehr als durch reinen Zufall möglich wäre, schreiben die Autoren. Für den Bereich Selektivität und Intensität der Aufmerksamkeit lag der Anteil positiver Studien mit 33-44\% noch höher (sieben- bis achtfach über dem Zufallsniveau). Auch für Handlungssteuerung und Intelligenz ließ sich ein signifikanter Anteil positiver Studien ausmachen. Am besten schnitt Ginkgo in den Bereichen fluide Intelligenz ( $38 \%$ positive Studien) und selektive Aufmerksamkeit (44\%) ab. Mehr als zwei Drittel der Studien wurden mit dem Extrakt EGb $761^{\circledR}$ durchgeführt.

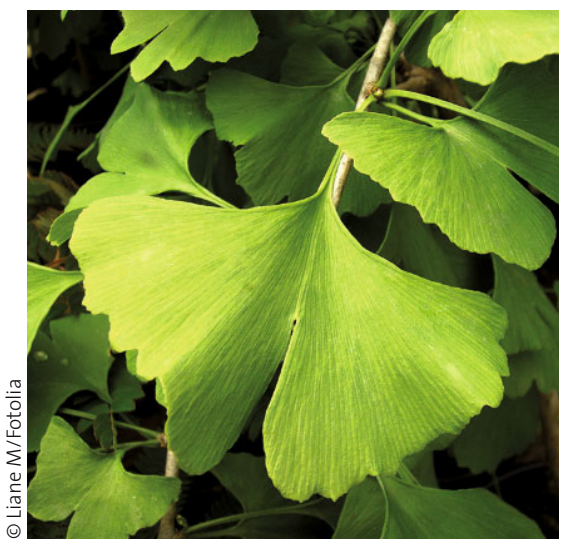

Ginkgo biloba: widersprüchliche Studiendaten.

Trotz aller methodischen Einschränkungen zeichnet sich $a b$, dass komplexe kognitive Funktionen wie Gedächtnis, Aufmerksamkeit und Intelligenz durch Ginkgo verbessert werden können, so die Autoren der Metaanalyse. Einiges spreche dafür, dass der Pflanzenextrakt in vielen Studien nicht sein volles Potenzial zeigen konnte (sogenannte ß-Fehler). Dazu gehörten beispielsweise zu „gesunde“ Probanden oder zu kurze Behandlungsdauer. Dies könnte die Heterogenität der Studienergebnisse zumindest teilweise erklären, so die Autoren.

- Maria Weiß

Quelle: JAMA 2008;300:2253; JAMA 2009;302:2663; Kaschel R et al; Human Pharmacopsychology 2009;24:345-370

\section{Lokaltherapie von Schmerzen am Bewegungsapparat Kleben statt schmieren}

- Zur Behandlung von Schmerzen bei orthopädischen Überlastungssyndromen eignet sich in vielen Fällen die (zusätzliche) lokale Applikation von NSAR. Ein Pflaster mit dem Wirkstoff DiclofenacEpolamin (Flector ${ }^{\circledR}$ Schmerzplaster) bietet dabei Vorteile gegenüber Diclofenac in Gelform.

Das Epolaminsalz von Diclofenac wird aus einer Polymermatrix über mindestens zwölf Stunden gleichmäßig freigesetzt. Damit hat das Pflaster eine längere Wirkdauer und sorgt länger für konstan- te Wirkspiegel als ein Diclofenac-Natrium-Gel. Das spiegeln auch klinische Studiendaten wider: Mit dem Pflaster wird eine bessere Schmerzreduktion erreicht als mit Diclofenac Emulgel.

In Deutschland ist das Pflaster bisher nur bei Sprunggelenksdistorsion und Epikondylitis zugelassen. In zwei Doppelblindstudien mit 134 bzw. 190 Patienten ergab sich in beiden Indikationen eine signifikante Überlegenheit gegenüber Placebo. Wie die Weiterbeobachtung der Epikondylitispatienten ergab, blieb ein signifikanter Unterschied auch noch 14 Tage über das Behandlungsende hinaus erhalten. In der Schweiz und den USA, wo das NSAR-Pflaster bereits seit vielen Jahren auf dem Markt ist, wird es auch in anderen Indikationen eingesetzt, u. a. bei Gonarthrose und bei entzündlichen Gelenk- und Muskelbeschwerden. Das Pflaster wird einmal am Tag gewechselt. Ein elastischer Netzstrumpf erleichtert die Fixation.

- Quelle: Jenoure P. J. Traumatol Sport 2003; IBSA, Schweiz 